\title{
Lie theory and the wave equation in space-time. 3. Semisubgroup coordinates
}

\author{
E. G. Kalnins \\ Mathematics Department, University of Waikato, Hamilton, New Zealand \\ W. Miller, Jr. \\ School of Mathematics, University of Minnesota, Minneapolis, Minnesota 55455 \\ (Received 22 March 1976)
}

\begin{abstract}
We classify and study those coordinate systems which permit $R$ separation of variables for the wave equation in four-dimensional space-time and such that at least one of the variables corresponds to a oneparameter symmetry group of the wave equation. We discuss over 100 such systems and relate them to orbits of triplets of commuting operators in the enveloping algebra of the conformal group $\operatorname{SO}(4,2)$.
\end{abstract}

\section{INTRODUCTION}

This paper is an introduction to the problem of $R$ separation of variables for the wave equation

$$
\left(\partial_{00}-\partial_{11}-\partial_{22}-\partial_{33}\right) \Psi(x)=0 .
$$

As is well known, ${ }^{1}$ the symmetry group of (1.1) is locally isomorphic to the fifteen-parameter group $S O(4,2)$. In this and subsequent papers we will show explicitly that every known separable coordinate system for (1.1) (as well as some systems which we derive for the first time) corresponds to a three-dimensional commuting subspace of the space of second-order elements in the enveloping algebra of so( 4,2$)$. [We consider the elements of so $(4,2)$ as first-order differential operators which map solutions of (1.1) into solutions.] If the commuting operators $S_{1}, S_{2}, S_{3}$ form a basis for such a subspace then the associated $R$-separable solutions $\Psi$ of $(1.1)$ are characterized by the eigenvalue equations $S_{j} \Psi=\lambda_{j} \Psi$, $j=1,2,3$, where the eigenvalues $\lambda_{j}$ are the separation constants. The group $\mathrm{SO}(4,2)$ acts on the enveloping algebra of so $(4,2)$ via the adjoint representation and decomposes the set of three-dimensional commuting subspaces of second-order elements into $\mathrm{SO}(4,2)$-orbits. We regard coordinate systems associated with subspaces on the same orbit as equivalent.

Several earlier papers of the authors and collaborators can be considered as preparation for the problem we tackle directly here. In particular, the Helmholtz, ${ }^{2}$ Klein-Gordon, ${ }^{3}$ and Euler-Poisson-Darboux, ${ }^{4}$ equations are special cases of (1.1) as are the eigenvalue equations for the Laplace operator on the sphere $S_{3}{ }^{5}$ and the hyperboloids of one and two sheets. ${ }^{6}$ The same is true for the time-dependent Schrödinger equations for the free-particle, free-fall, harmonic oscillator ${ }^{7}$ and hydrogen atom. Our procedure will follow closely the analogous study of the three-variable wave equation in Refs. 3, 8, and 9. The difference consists mainly in the greater complexity of the four-variable case (although a number of computations turn out to be easier in four dimensions than in three). In this paper we proceed as in Ref. 8 and present a group theoretic analysis of (1.1) as well as a rough classification of semisubgroup systems for this equation. Our future (much more detailed) results will be fitted into the framework established here.
In Sec. 2 we define the symmetry algebra so $(4,2)$ of (1.1) in two distinct bases and construct a Fouriertransform Hilbert space $H$ as well as a Hilbert space of positive energy solutions of this equation. On $H$, the elements of so $(4,2)$ exponentiate to yield a unitary irreducible representation of the covering group $\mathrm{SU}(2,2)$ of the identity component in $\operatorname{SO}(4,2)$. In Sec. 3 we determine explicitly the action of $\operatorname{SU}(2,2)$. Most of the results in this section were obtained in Ref. 10 by another method. [However, Eq. (6.6) for the action of a lightlike special conformal transformation appears to be new. ]

The remainder of the paper is concerned with separation of variables. In analogy with Ref. 8 we say that $R-$ separable coordinates $\left\{u_{j}\right\}$ associated with a threedimensional commuting subspace of symmetry operators are semisubgroup coordinates if the subspace has a basis $S_{1}, S_{2}, S_{3}$ such that $S_{1}=A^{2}$ where $A \in \operatorname{so}(4,2)$ and $\left[A, S_{j}\right]$ $=0, j=2$, 3. A particular $A \in \mathrm{so}(4,2)$ may correspond to several (or to no) semisubgroup systems. If $\Psi$ satisfies (1.1) and the equation $A \Psi=i \lambda \Psi$, then, since $A$ is a symmetry of (1.1), we can use standard Lie theory and introduce new variables $y_{0}, y_{1}, y_{2}, y_{3}$ such that $A=\partial_{y_{0}}+f(y)$ and $\Psi(y)=r(y) \exp \left(i \lambda y_{0}\right) \Phi_{\lambda}\left(y_{j}\right)$, where $r$ is a fixed function satisfying $\partial_{y_{0}} r+f r=0$. Then (1.1) reduces to a second-order partial differential equation $(\dagger)$ for $\Phi_{\lambda}$ in the three variables $y_{j}$. The possible semisubgroup systems $A^{2}, S_{2}, S_{3}$ thus correspond to the possible coordinate systems such that the reduced equation $(\dagger)$ separates.

In Secs. $4-8$ we examine the possible semisubgroup systems for which $S_{2}$ and $S_{3}$ belong to the symmetry enveloping algebra of $(\dagger)$. They are of seven types corresponding to seven choices for $A$. Using the notation introduced in Sec. 2, we find the types are:

1] $A=\Gamma_{56}$. In this case ( $\left.\dagger\right)$ is the eigenvalue equation for the Laplace operator on the sphere $S_{3}$. There are six coordinate systems. ${ }^{5}$

2] $A=P_{0}$ and $(\dagger)$ is the Helmholtz equation (5.1) which separates in 11 coordinate systems. ${ }^{2}$

3] $A=P_{3}$ and ( $\dagger$ ) is the Klein-Gordon equation (5.4) which separates in 53 orthogonal coordinate systems. ${ }^{3}$

4] $A=D$ and $(\dagger)$ is the eigenvalue equation (5.8) for 
the Laplace operator on the hyperboloid which separates in 35 coordinate systems. ${ }^{6}$

5] $A=P_{0}+P_{1}$ and $(\dagger)$ is the free particle Schrödinger equation (6.1). There are 17 coordinate systems. ${ }^{7}$

6] $A=\Gamma_{43}$ and ( $\dagger$ ) is a generalized EPD equation (7.1). The number of coordinate systems for this case has not yet been determined.

7] $A=\Gamma_{12}+\Gamma_{34}-\Gamma_{56}$ and $(\dagger)$ is (8.3). The number of coordinate systems for this case is still unknown.

Cases 6] and 7] will be discussed in detail in future papers. For each case we show how to pass from the Fourier-transform Hilbert space $H$ to the space of positive energy solutions of (1.1).

Our classification includes all known semisubgroup coordinates for (1.1) with one principal exception. Diagonalization of the operator $A=P_{0}+H_{1}$ in Case 5] does not uniquely determine the variable $y_{0}$ which is split off to obtain the reduced equation $(t)$. Thus $(t)$ is not unique in this case. The possibilities for the nonorthogonal coordinates which can arise will be classified in a future paper. [See Ref. 9 where a similar classification was carried out for $\left(\partial_{t t}-\partial_{x x}-\partial_{y y}\right) \Psi=0$. ] In all other cases there is an identity analogous to (2.24) which uniquely determines the reduced equation. The variable $y_{0}$ is still not unique, but new nonorthogonal coordinates so obtained are rather trivial modifications of the coordinates we have listed.

Finally, in Sec. 9 we classify the orbits in so(4,2) under the adjoint action of $\mathrm{SO}(4,2)$ to see why not every $A \in$ so $(4,2)$ belongs to a semisubgroup system.

The next two papers in this series will be devoted to an explicit classification of all orthogonal $R$-separable coordinate systems (semisubgroup or not) whose coordinate surfaces are families of confocal cyclides. The classification will proceed in analogy to that in Ref. 3. However, the number of coordinate systems involved is approximately 300 . Later we will classify the nonorthogonal systems. Future work will concern the results in special function theory which follow from separation of variables in (1.1). Equation (1.1) is the most important equation in special function theory and it is no accident that Bateman ${ }^{11,12}$ devoted so much energy to its solution by separation of variable methods.

\section{SO(4, 2) AND THE WAVE EQUATION}

The symmetry algebra of the wave equation

$$
\left(\partial_{00}-\partial_{11}-\partial_{22}-\partial_{33}\right) \Psi(x)=0, \quad x=\left(x_{0}, x_{1}, x_{2}, x_{3}\right)
$$

is the set of all linear differential operators

$$
L=\sum_{j=0}^{3} a_{j}(x) \partial_{j}+b(x)
$$

such that $L \Psi$ is a (local) solution of (1.1) whenever $\Psi$ is a (local) solution.

As is well known, the possible symmetry operators $L$ form a 15 -dimensional Lie algebra, isomorphic to so $(4,2)$, where the commutator is the usual Lie bracket. ${ }^{13}$ A convenient basis for this model of so $(4,2)$ is provided by the linear momentum operators

$$
P_{\alpha}=\partial_{\alpha}, \quad \alpha=0,1,2,3,
$$

the generators of homogeneous Lorentz transformations

$$
\begin{aligned}
& M_{21}=x_{2} \partial_{1}-x_{1} \partial_{2}, \quad M_{13}=x_{1} \partial_{3}-x_{3} \partial_{1}, \quad M_{32}=x_{3} \partial_{2}-x_{2} \partial_{3}, \\
& M_{01}=x_{01} \partial_{1}+x_{1} \partial_{01}, \quad M_{02}=x_{0} \partial_{2}+x_{2} \partial_{0}, \quad M_{03}=x_{0} \partial_{3}+x_{3} \partial_{0}, \\
& M_{j k}=-M_{k j}, \quad M_{0 j}=M_{j 0}, \quad j, k=1,2,3,
\end{aligned}
$$

the generator of dilatations

$$
D=-\left(1+x_{0} \partial_{0}+x_{1} \partial_{1}+x_{2} \partial_{2}+x_{3} \partial_{3}\right),
$$

and the generators of special conformal transformations

$$
\begin{aligned}
& K_{0}=-2 x_{0}+\left(x \cdot x-2 x_{0}^{2}\right) \partial_{0}-2 x_{0} x_{1} \partial_{1}-2 x_{0} x_{2} \partial_{2}-2 x_{0} x_{3} \partial_{3}, \\
& K_{1}=2 x_{1}+\left(x \cdot x+2 x_{1}^{2}\right) \partial_{1}+2 x_{1} x_{0} \partial_{0}+2 x_{1} x_{2} \partial_{2}+2 x_{1} x_{3} \partial_{3}, \\
& K_{2}=2 x_{2}+\left(x \cdot x+2 x_{2}^{2}\right) \partial_{2}+2 x_{2} x_{0} \partial_{0}+2 x_{2} x_{1} \partial_{1}+2 x_{2} x_{3} \partial_{3}, \\
& K_{3}=2 x_{3}+\left(x \cdot x+2 x_{3}^{2}\right) \partial_{3}+2 x_{3} x_{0} \partial_{0}+2 x_{3} x_{1} \partial_{1}+2 x_{3} x_{2} \partial_{2},
\end{aligned}
$$

where

$$
\begin{aligned}
x \cdot y & =x_{0} y_{0}-x_{1} y_{1}-x_{2} y_{2}-x_{3} y_{3} \\
& =x_{0} y_{0}-\mathrm{x} \cdot \mathrm{y}=\sum_{\alpha \beta=0}^{3} g_{\alpha \beta} x_{\alpha} y_{B} .
\end{aligned}
$$

The commutation relations will follow from relations (2.22) to be derived later.

The symmetry operators can be exponentiated to yield a local Lie transformation group of symmetries of (2.1). ${ }^{13,14}$ Indeed, the momentum and Lorentz operators generate the Poincaré group of symmetries

$$
\Psi(x) \rightarrow \Psi\left(\Lambda^{-1}(x-a)\right), \quad a=\left(a_{0}, a_{1}, a_{2}, a_{3}\right), \quad \Lambda \in \mathrm{SO}(1,3),
$$

while the dilatation operators generate

$$
\exp (\lambda D) \Psi(x)=\exp (-\lambda) \Psi(\exp (-\lambda) x), \quad \lambda \in R
$$

and the $K_{\alpha}$ generate the special conformal tranformations

$$
\begin{aligned}
& \exp \left(a_{0} K_{0}+a_{1} K_{1}+a_{2} K_{2}+a_{3} K_{3}\right) \Psi(x) \\
& =[1+2 x \cdot a+(a \cdot a)(x \cdot x)]^{-1} \Psi\left(\frac{x+a(x \cdot x)}{1+2 x \cdot a+(a \cdot a)(x \cdot x)}\right) .
\end{aligned}
$$

We shall also consider the inversion operator

$$
R \Psi(x)=(x \cdot x)^{-1} \Psi(-x / x \cdot x)
$$

which is a symmetry not generated by the local Lie symmetries (2.2)-(2.5).

As is well known from quantum field theory, ${ }^{1,13}$ by formally taking the Fourier transform in the variables $x_{\alpha}$ we can express the positive energy solutions of (2.1) in the form

$$
\begin{aligned}
& \Psi(x)=\frac{1}{(2 \pi)^{3 / 2}} \iiint_{-\infty}^{\infty} \exp (i k \cdot x) f(\mathbf{k}) d \mu(\mathbf{k}) \\
& k_{0}=\left(k_{1}^{2}+k_{2}^{2}+k_{3}^{2}\right)^{1 / 2}, \quad d \mu(\mathrm{k})=d k_{1} d k_{2} d k_{3} / k_{0} .
\end{aligned}
$$

Let $H$ be the Hilbert space of all complex Lebesgue measurable functions $f(\mathrm{k})$ such that

$$
\iiint_{-\infty}^{\infty}|f|^{2} d \mu(\mathbf{k})<\infty,
$$

and with inner product 


$$
\langle f, g\rangle=\iiint f \bar{g} d \mu(\mathbf{k}), \quad f, g \in H .
$$

As is well known, ${ }^{1,13}$ the functions $\Psi, \Phi$ related to $f, g$, respectively, by (2.11) satisfy

$$
\begin{aligned}
\langle\Psi, \Phi\rangle & \equiv\langle f, g\rangle=i \iint_{x_{0}=t} \int_{t} \Psi(x) \partial_{0} \bar{\Phi}(x) d x_{1} d x_{2} d x_{3} \\
& =-i \iint_{x_{0}=t} \int\left(\partial_{0} \Psi(x)\right) \bar{\Phi}(x) d x_{1} d x_{2} d x_{3},
\end{aligned}
$$

independent of $t$. [Note that (2.14) is easily derived from (2.13) for $f, g$ belonging to the dense subspace $D$ of $H$ consisting of $C^{\infty}$ functions with compact support bounded away from $\mathbf{k}=0$, and then considering the closure of $D$. For $f \in H$ the corresponding $\Psi$ is a solution of (2.1) in the sense of distribution theory.]

The operators (2.2)-(2.5) acting on solutions of (2.1) induce, via (2.11), corresponding operators on $H$,

$$
\begin{aligned}
P_{0}= & i k_{0}, \quad P_{j}=-i k_{j}, \quad j=1,2,3, \\
M_{21}= & k_{2} \partial_{k_{1}}-k_{1} \partial_{k_{2}}, \quad M_{13}=k_{1} \partial_{k_{3}}-k_{3} \partial_{k_{1}}, \\
M_{32}= & k_{2} \partial_{k_{3}}-k_{3} \partial_{k_{2}}, \quad M_{01}=k_{0} \partial_{k_{1}}, \\
M_{02}= & k_{0} \partial_{k_{2}}, \quad M_{03}=k_{0} \partial_{k_{3}} \\
D=1 & +k_{1} \partial_{k_{1}}+k_{2} \partial_{k_{2}}+k_{3} \partial_{k_{3}}, \\
K_{0}= & i k_{0}\left(\partial_{k_{1} k_{1}}+\partial_{k_{2} k_{2}}+\partial_{k_{3} k_{3}}\right) \\
K_{1}= & i\left(k_{1} \partial_{k_{1} k_{1}}-k_{1} \partial_{k_{2} k_{2}}-k_{1} \partial_{k_{3} k_{3}}\right. \\
& \left.+2 k_{2} \partial_{k_{1} k_{2}}+2 k_{3} \partial_{k_{1} k_{3}}+2 \partial_{k_{1}}\right) \\
K_{2}= & i\left(k_{2} \partial_{k_{2} k_{2}}-k_{2} \partial_{k_{1} k_{1}}-k_{2} \partial_{k_{3} k_{3}}\right. \\
& \left.+2 k_{1} \partial_{k_{2} k_{1}}+2 k_{3} \partial_{k_{2} k_{3}}+2 \partial_{k_{2}}\right) \\
K_{3}= & i\left(k_{3} \partial_{k_{3} k_{3}}-k_{3} \partial_{k_{1} k_{1}}-k_{3} \partial_{k_{2} k_{2}}\right. \\
& \left.+2 k_{1} \partial_{k_{3} k_{1}}+2 k_{2} \partial_{k_{3} k_{2}}+2 \partial_{k_{3}}\right) .
\end{aligned}
$$

In Ref. 13 it is shown that $H$ is invariant under $R$ and

$$
R f(\mathbf{k})=\frac{1}{4 \pi} \iiint J_{0}\left((2 k \cdot l)^{1 / 2}\right) f(\mathbf{l}) d \mu(\mathbf{l}),
$$

where $J_{0}(z)$ is a Bessel function. Furthermore, $R^{2}=E$ (the identity operator on $f$ ) and $R$ is a unitary selfadjoint operator on this space. Also we have the relations

$$
R K_{\alpha} R^{-1}=-P_{\alpha}, \quad R D R^{-1}=-D, \quad R M_{\alpha \beta} R^{-1}=M_{\alpha \beta} .
$$

Note: There is a minus sign error in the corresponding expression in Ref. 8 which propagates through several equations. The error is corrected in Ref. 15.

Now we introduce a new basis for the symmetry algebra of (2.1) which makes apparent the isomorphism with so $(4,2)$. We define $s o(4,2)$ as the 15 -dimensional Lie algebra of $6 \times 6$ real matrices $A$ such that $A G+G A^{t}=0$, where 0 is the zero matrix and

$$
G=\left[\begin{array}{rrrrr}
1 & & & & 0 \\
& 1 & & & \\
& & 1 & & \\
& & 1 & & \\
& & & -1 & \\
0 & & & & -1
\end{array}\right]=\left(G_{\alpha \beta}\right) .
$$

Let $\mathcal{E}_{\text {if }}$ be the $6 \times 6$ matrix with a 1 in row $i$, column $j$, and zeros everywhere else. It is straightforward to check that the matrices

$$
\begin{aligned}
& \Gamma_{a b}=\varepsilon_{a b}-\varepsilon_{b a}=-\Gamma_{b a}, \quad 1 \leqslant a<b \leqslant 4, \\
& \Gamma_{a A}=\varepsilon_{a A}+\varepsilon_{A a}=-\Gamma_{A a}, \quad 1 \leqslant a \leqslant 4, \quad A=5,6, \\
& \Gamma_{56}=\varepsilon_{65}-\varepsilon_{56}=-\Gamma_{65}
\end{aligned}
$$

form a basis for $\operatorname{so}(4,2)$ with commutation relations

$$
\begin{aligned}
{\left[\Gamma_{\alpha \beta}, \Gamma_{\gamma \varphi}\right]=} & G_{\beta \gamma} \Gamma_{\alpha \varphi}+G_{\alpha \varphi} \Gamma_{\beta \gamma}-G_{\varphi \beta} \Gamma_{\alpha \gamma} \\
& -G_{\alpha \gamma} \Gamma_{\beta \varphi}
\end{aligned}
$$

This basis can be related to the operators (2.2)-(2.5) as follows:

$$
\begin{array}{lll}
P_{0}=\Gamma_{15}+\Gamma_{56}, & P_{1}=\Gamma_{12}+\Gamma_{26}, & P_{2}=\Gamma_{13}+\Gamma_{36}, \\
P_{3}=\Gamma_{14}+\Gamma_{46}, & K_{0}=\Gamma_{15}-\Gamma_{56}, & K_{1}=\Gamma_{12}-\Gamma_{26}, \\
K_{2}=\Gamma_{13}-\Gamma_{36}, & K_{3}=\Gamma_{14}-\Gamma_{46}, & M_{21}=\Gamma_{32}, \\
M_{13}=\Gamma_{24}, & M_{32}=\Gamma_{43}, \quad M_{01}=\Gamma_{25}, \\
M_{02}=\Gamma_{35}, & M_{03}=\Gamma_{45}, \quad D=\Gamma_{16} .
\end{array}
$$

(That is, the appropriate commutation relations are satisfied if these identifications are made.)

For our models of so(4,2), [acting on the solution space of (2.1) or on the Hilbert space $H]$ we have the identities

$$
\begin{aligned}
& \text { (i) } P_{0}^{2}-P_{1}^{2}-P_{2}^{2}-H_{3}^{2}=K_{0}^{2}-K_{1}^{2}-K_{2}^{2}-K_{3}^{2}=0, \\
& \text { (ii) } \Gamma_{12}^{2}+\Gamma_{13}^{2}+\Gamma_{14}^{2}+\Gamma_{23}^{2}+\Gamma_{24}^{2}+\Gamma_{34}^{2}=\Gamma_{56}^{2}+1, \\
& \text { (iii) } \Gamma_{32}^{2}+\Gamma_{24}^{2}+\Gamma_{43}^{2}-\Gamma_{25}^{2}-\Gamma_{35}^{2}-\Gamma_{45}^{2}=-D^{2}+1, \\
& \text { (iv) } \Gamma_{12}^{2}+\Gamma_{56}^{2}-\Gamma_{15}^{2}-\Gamma_{16}^{2}-\Gamma_{26}^{2}-\Gamma_{25}^{2}=\Gamma_{43}^{2}+1
\end{aligned}
$$

If $\left\{\Psi_{\alpha}(x)\right\}$ is an orthonormal (ON) basis for the Hilbert space of positive energy solutions of $(2.1)$ then (in the sense of distributions)

$$
\begin{aligned}
\sum_{\alpha} \Psi_{\alpha}(x) \Psi_{\alpha}\left(x^{\prime}\right)= & \Delta_{+}\left(x-x^{\prime}\right)=\frac{1}{(2 \pi)^{3}} \iiint \\
& \times \exp \left[i k \cdot\left(x-x^{\prime}\right)\right] d \mu(\mathbf{k}),
\end{aligned}
$$

where the distribution $\Delta_{+}$is given explictly by ${ }^{16}$

$$
\begin{aligned}
& \Delta_{+}(x)=\frac{1}{(2 \pi)^{2}} \frac{1}{r^{2}-t^{2}}+\frac{i}{4 \pi r}[\delta(r+t)-\delta(r-t)], \\
& r=\left(x_{1}^{2}+x_{2}^{2}+x_{3}^{2}\right)^{1 / 2}, \quad t=x_{0} .
\end{aligned}
$$

Note that

$$
\Psi(x)=\left\langle\Psi, \Delta_{+}\left(x^{\prime}-x\right)\right\rangle,
$$

where the integration is carried out over $\mathbf{x}^{\prime}$.

\section{THE ACTION OF THE CONFORMAL GROUP}

As is well known, the representation of so $(4,2)$ on $H$ defined by the operators $(2.15)-(2.18)$ can be extended to a unitary irreducible representation of the covering group $S U(2,2)$ of the identity component of $S O(4,2) .^{10}$ The maximal compact connected subgroup of $\mathrm{SU}(2,2)$ is $S O(4) \times S O(2)$, where $S O(4)$ is generated by the Lie algebra operators $\Gamma_{i j}, 1 \leqslant i<j \leqslant 4$ and $\mathrm{SO}(2)$ by $\Gamma_{56}$. We will explicitly determine the action of this subgroup on $H$ as well as the actions of other interesting subgroups. 
The operators $M_{\alpha \beta}$ generate a subgroup of $\mathrm{SU}(2,2)$ isomorphic to the homogeneous Lorentz group $\mathrm{SO}(3,1)$. The action of this subgroup is determined by

$$
\begin{gathered}
T(0) f(\mathbf{k})=f(\mathbf{k} 0), \quad 0 \in \mathrm{SO}(3), \\
\exp \left(a M_{01}\right) f(\mathbf{k})=f\left(k_{1}(a), k_{2}, k_{3}\right), \\
k_{1}(a)=k_{1} \cosh a+k_{0} \sinh a,
\end{gathered}
$$

where $M_{21}, M_{13}, M_{32}$ generate $\mathrm{SO}(3)$, and the results for $M_{02}, M_{03}$ follow easily from that for $M_{01}$. The $P_{\alpha}$ generate a translation subgroup of operators

$$
\exp \left(\Sigma a_{\alpha} P_{\alpha}\right) f(\mathrm{k})=\exp (i a \cdot k) f(\mathrm{k}) .
$$

The unitary operators $\exp \left(\sum a_{\alpha} K_{\alpha}\right)$ are more difficult to compute. Since the $\mathrm{SO}(3,1)$ subgroup transforms $a$ via the adjoint action, one has to consider only three distinct cases : (1) $a=\left(a_{0}, 0,0,0\right), a_{0} \neq 0$, timelike; (2) $a=\left(0, a_{1}, 0,0\right), a_{1} \neq 0$, spacelike; (3) $a=\left(a_{1}, a_{1}, 0,0\right)$, $a_{1} \neq 0$, lightlike. All other cases can be obtained by composing these three operators with the operators (3.1), (3.2).

Starting with the timelike case, we introduce the basis $\left\{f_{l_{j}}\right\}$ for $H$ consisting of generalized eigenvectors of the commuting operators $P_{\alpha}$,

$$
\begin{aligned}
& f_{l_{j}}(\mathrm{k})=\delta\left(k_{1}-l_{1}\right) \delta\left(k_{2}-l_{2}\right) \delta\left(k_{3}-l_{3}\right) k_{0}, \quad-\infty<l_{j}<\infty \\
& P_{h} f_{l_{j}}=-i l_{h} f_{l_{j}}, \quad h=1,2,3, \quad P_{0} f_{l_{j}}=i l_{0} f_{l_{j}}, \\
& \left\langle f_{l_{j}}, f_{l_{j}}\right\rangle=\delta\left(l_{1}-l_{1}^{\prime}\right) \delta\left(l_{2}-l_{2}^{\prime}\right) \delta\left(l_{3}-l_{3}^{\prime}\right) l_{0}, \\
& l_{0}=\left(l_{1}^{2}+l_{2}^{2}+l_{3}^{2}\right)^{1 / 2} .
\end{aligned}
$$

It follows that the functions $g_{l_{j}}=R f_{l_{j}}$,

$$
g_{l_{j}}(\mathbf{k})=\frac{1}{4 \pi} J_{0}\left((2 k \cdot l)^{1 / 2}\right),
$$

form a basis for $H$ consisting of generalized eigenvectors of the commuting operators $K_{\alpha}$,

$$
\begin{aligned}
& K_{h g_{l_{j}}}=i l_{h} g_{l_{j}}, \quad K_{0} g_{l_{j}}=-i l_{0} g_{l_{j}}, \\
& \left\langle g_{l_{j}}, g_{l^{\prime}}\right\rangle=\delta\left(l_{1}-l_{1}^{\prime}\right) \delta\left(l_{2}-l_{2}^{\prime}\right) \delta\left(l_{3}-l_{3}^{\prime}\right) l_{0},
\end{aligned}
$$

as follows from the fact that $R$ is unitary.

Now we have for $f \in H$ that

$$
\begin{aligned}
& \exp \left(a K_{0}\right) f(\mathbf{s})=\iiint G(a, 1, \mathbf{s}) f(\mathbf{l}) d \mu(1) \\
& G(a, \mathbf{l}, \mathbf{s})=\left\langle\exp \left(a K_{0}\right) f_{l_{j}}, f_{s_{j}}\right\rangle \\
&=\left\langle R \exp \left(-a P_{0}\right) R f_{l_{j}}, f_{s_{j}}\right\rangle=\left\langle\exp \left(-a P_{0}\right) g_{l_{j}}, g_{s_{j}}\right\rangle \\
&= \frac{1}{16 \pi^{2}} \iiint \exp \left(-i a k_{0}\right) J_{0}\left((2 k \cdot l)^{1 / 2}\right) d \mu(\mathbf{k}) \\
&= \frac{-1}{4 \pi|a|} \exp \left[i\left(l_{0}+s_{0}\right) a^{-1}\right] \\
& \times J_{0}\left(a^{-1}\left[2\left(s_{0} l_{0}+s_{1} l_{1}+s_{2} l_{2}+s_{3} l_{3}\right)\right]^{1 / 2}\right),
\end{aligned}
$$

$a \neq 0$.

To compute the action of $\exp \left(a K_{1}\right)$, we choose a basis of eigenfunctions of the commuting operators $P_{0}, P_{1}, M_{32}$,

$$
\begin{aligned}
& h_{p \lambda m}(\mathbf{k})=(2 \pi)^{-1 / 2} \delta\left(k_{0}-\rho\right) \delta\left(k_{1}-\lambda\right) \exp (i m \theta), \\
& 0 \leqslant \rho, \quad-\rho \leqslant \lambda \leqslant \rho, \quad m=0, \pm 1, \ldots, \\
& d \mu(\mathbf{k})=d k_{0} d k_{1} d \theta, \quad k_{2}=\left(k_{0}^{2}-k_{1}^{2}\right)^{1 / 2} \sin \theta,
\end{aligned}
$$

Here,

$$
k_{3}=\left(k_{0}^{2}-k_{1}^{2}\right)^{1 / 2} \cos \theta .
$$

$$
\begin{aligned}
& P_{0} h=i \rho h, \quad P_{1} h=-i \lambda h, \quad M_{32} h=i m h, \\
& \left\langle h_{\rho \lambda m_{z}} h_{\rho^{\prime} \lambda^{\prime} m^{\prime}}\right\rangle=\delta\left(\rho-\rho^{\prime}\right) \delta\left(\lambda-\lambda^{\prime}\right) \delta_{m m^{\circ}}
\end{aligned}
$$

It follows that the functions $g_{\rho \lambda m}=R h_{p \lambda m}$,

$$
\begin{aligned}
& g_{\rho \lambda m}(\mathbf{k})=\frac{\exp (i m \varphi)}{(8 \pi)^{1 / 2}} J_{m}\left[(r s \exp (\alpha-\beta))^{1 / 2}\right] \\
& \quad \times J_{m}\left[(r s \exp (\beta-\alpha))^{1 / 2}\right] \\
& \rho=r \cosh \alpha, \quad \lambda=r \sinh \alpha, \\
& k_{0}=s \cosh \beta, \quad k_{1}=s \sinh \beta, \\
& d \mu(\mathbf{k})=s d s d \beta d \theta, \quad s \geqslant 0, \quad-\infty<\beta<\infty, \quad-\pi \leqslant \theta \leqslant \pi,
\end{aligned}
$$

form a basis for $f i$ and satisfy relations

$$
\begin{aligned}
& K_{0} g=-i \rho g, \quad K_{1} g=i \lambda g, \quad M_{32} g=i m g, \\
& \left\langle g_{\rho \lambda m}, g_{\rho^{\prime} \lambda m^{\circ}}\right\rangle=\delta\left(\rho-\rho^{\prime}\right) \delta\left(\lambda-\lambda^{\prime}\right) \delta_{m m m^{\circ}}
\end{aligned}
$$

Now,

$$
\exp \left(a K_{1}\right) f(\mathbf{k})=\int H(a, \mathbf{l}, \mathbf{k}) f(\mathbf{l}) d \mu(\mathbf{l}),
$$

where

$$
\begin{aligned}
H(a, \mathbf{1}, \mathbf{k})= & \left\langle\exp \left(a K_{1}\right) f_{\mathbf{1}}, f_{\mathbf{k}}\right\rangle \\
= & \sum_{m=-\infty}^{\infty} \int_{0}^{\infty} \int_{-\infty}^{\rho}\left\langle f_{\mathbf{1}}, \exp \left(-a K_{1}\right) g_{0 \lambda m}\right\rangle\left\langle g_{\rho \lambda m}, f_{\mathbf{k}}\right\rangle d \lambda d \rho \\
= & \sum_{m=-\infty}^{\infty} \int_{0}^{\infty} \int_{-\rho}^{\rho} \exp (i \lambda a) \overline{g_{\rho \lambda m}}(\mathbf{1}) g_{\rho \lambda m}(\mathbf{k}) d \lambda d \rho \\
= & \frac{1}{4 \pi a^{2}} \exp \left[i\left(k_{1}+l_{1}\right) / a\right] \\
& \times J_{0}\left(a^{-1} \sqrt{2}\left(k_{0} l_{0}+k_{1} l_{1}-k_{2} l_{2}-k_{3} l_{3}\right)^{1 / 2}\right) .
\end{aligned}
$$

We will compute expa $\left(K_{0}+K_{1}\right)$ in Sec。 6 .

The dilatation operator generates the symmetries

$$
\exp (a D) f(\mathbf{k})=e^{a} f\left(e^{a} \mathbf{k}\right) .
$$

Using these results we can exponentiate the compact generator $\Gamma_{56}=\frac{1}{2}\left(F_{0}-K_{0}\right)$. The operators $P_{0}, D, K_{0}$ generate a $\mathrm{SL}(2, R)$ subgroup of $\mathrm{SU}(2,2)$ and we have

$$
\begin{aligned}
\exp \left(2 \theta \Gamma_{56}\right)= & \exp \left(\tan \theta P_{0}\right) \exp \left(-\sin \theta \cos \theta K_{0}\right) \\
& \times \exp (-2 \ln \cos \theta D)
\end{aligned}
$$

on $\operatorname{SL}(2, R)$. Evaluating the right-hand side of this expression we find

$$
\begin{aligned}
\exp \left(2 \theta \Gamma_{56}\right) f(\mathbf{k}) \\
=-\frac{|\cot \theta|}{4 \pi} \int \exp \left[-i\left(l_{0}+k_{0}\right) \cot \theta\right] J_{0}\left(\operatorname { c s c } \theta \left[2 \left(k_{0} l_{0}+k_{1} l_{1}\right.\right.\right. \\
\left.\left.\left.\quad+k_{2} l_{2}+k_{3} l_{3}\right)\right]^{1 / 2}\right) f(1) d \mu(1), \quad \theta \neq n \pi .
\end{aligned}
$$

The operators $P_{1}, D, K_{1}$ generate another $\mathrm{SL}(2, R)$ subgroup of $\mathrm{SU}(2,2)$ and there follows the relation $\exp \left(2 \theta \Gamma_{12}\right)=\exp \left(\tan \theta P_{1}\right) \exp \left(\sin \theta \cos \theta K_{1}\right) \exp (-2 l n \cos \theta D)$ or

$$
\begin{aligned}
\exp \left(2 \theta \Gamma_{12}\right) & \\
= & \frac{1}{4 \pi \sin ^{2} \theta} \int \exp \left[i\left(k_{1}+l_{1}\right) \cot \theta\right\} J_{0}\left(\operatorname { c s c } \theta \left[2 \left(k_{0} l_{0}+k_{1} l_{1}\right.\right.\right. \\
& \left.\left.\left.-k_{2} l_{2}-k_{3} l_{3}\right)\right]^{1 / 2}\right) f(1) d \mu(1), \quad \theta \neq n \pi
\end{aligned}
$$




\section{DIAGONALIZATION OF $\Gamma_{56}$}

The restriction of the unitary irreducible representation $T$ of SU(2,2) on $H$, discussed above, to the compact subgroup $\mathrm{SO}(4)$ decomposes into a direct sum of SO(4)-irreducible representation $D_{F}, \operatorname{dim} D_{F}=(2 F+1)^{2}$. A basis of eigenvectors for the commuting operators $\Gamma_{56}, \Gamma_{43}, \Gamma_{12}$ can be used to exhibit this decomposition,

$$
\begin{aligned}
& \Gamma_{56} f=i \lambda f, \quad \Gamma_{43} f=i m f, \quad \Gamma_{12} f=i \rho f, \\
&-i \Gamma_{56}= \frac{1}{2} k_{0}\left(-\Delta_{3}+1\right), \quad \Gamma_{43}=k_{3} \partial_{k_{2}}-k_{2} \partial_{k_{3}}, \\
&-i \Gamma_{12}= \frac{k_{1}}{2}\left(\partial_{k_{1} k_{1}}-\partial_{k_{2} k_{2}}-\partial_{k_{3} k_{3}}-1\right) \\
&+k_{2} \partial_{k_{1} k_{2}}+k_{3} \partial_{k_{1} k_{3}}+\partial_{k_{1}} .
\end{aligned}
$$

Setting

$$
k_{1}=\left(\xi^{2}-\eta^{2}\right) / 2, \quad k_{2}=\xi \eta \sin \theta
$$

$$
\begin{aligned}
2 \Gamma_{16} f_{F a b}= & {[(F-a+1)(F+b+1)]^{1 / 2} f_{F+1 / 2, a-1 / 2, b+1 / 2}-[(F-a)(F+b)]^{1 / 2} f_{F+1 / 2, a+1 / 2, b-1 / 2} } \\
+ & {[(F+a+1)(F-b+1)]^{1 / 2} f_{F+1 / 2, a+1 / 2, b-1 / 2}-[(F+a)(F-b)]^{1 / 2} f_{F-1 / 2, a-1 / 2, b+1 / 2}, } \\
2 i P_{3} f_{F, a, b}= & {[(F+a+1)(F+b+1)]^{1 / 2} f_{F+1 / 2, a+1 / 2, b+1 / 2}-[(F-b)(F+b+1)]^{1 / 2} f_{F, a, b+1}-[(F-a)(F+a+1)]^{1 / 2} f_{F, a+1, b} } \\
& +[(F-a)(F-b)]^{1 / 2} f_{F-1 / 2, a+1 / 2, b+1 / 2}+[(F+a)(F+b)]^{1 / 2} f_{F-1 / 2, a-1 / 2, b-1 / 2}-[(F+b)(F-b+1)]^{1 / 2} f_{F, a, b-1} \\
& -[(F+a)(F-a+1)]^{1 / 2} f_{F, a-1, b}+[(F-a+1)(F-b+1)]^{1 / 2} f_{F+1 / 2, a-1 / 2, b-1 / 2 .}
\end{aligned}
$$

Expressions (4.1), (4.3), and the commutation relations (2.22) suffice to determine the action of any $\Gamma_{\alpha \beta}$ on $f_{F a b}$.

There is a close connection between the quantum Kepler problem in three-space,

$$
\begin{aligned}
& H \Phi=E \Phi, \quad H=-\partial_{x_{1} x_{1}}-\partial_{x_{2} x_{2}}-\partial_{x_{3} x_{3}}+e / r, \\
& r=\left(x_{1}^{2}+x_{2}^{2}+x_{3}^{2}\right)^{1 / 2}, \quad \iiint_{R^{3}}|\Phi|^{2} d x_{1} d x_{2} d x_{3}<\infty,
\end{aligned}
$$

and the equation $\Gamma_{56} f=i \lambda f$. Indeed the equations can be identified if we set $k_{j}=x_{j} \sqrt{-E}, E=-e^{2} / 4 \lambda^{2}$. (Although the eigenvalue problems are defined on Hilbert spaces with different inner products, it follows from the Virial theorem, Ref. 17 , p. 51 , that if $E$ belongs to the point spectrum of $H$, and $\Phi$ is the corresponding eigenvector, then $\Phi$ has finite norm in $H$. Conversely, if $f$ is an eigenvector of $\Gamma_{56}$ then $\iiint_{R^{3}}|f|^{2} d x_{1} d x_{2} d x_{3}<\infty$ and $f$ corresponds to an energy eigenvalue $E$ in the point spectrum of $H_{0}$ ) Since the eigenvalues of $-i \Gamma_{56}$ are $\lambda=2 F+1$, $2 F=0,1,2 \cdots$, it follows that the point spectrum of $H$ consists of the eigenvalues $E=-e^{2} / 4(2 F+1)^{2}$. (Similarly, the continuous spectrum of $H$ is related to the operator $\Gamma_{15}$.)

Applying the transformation (2.11) to the basis $\left\{f_{F a b}\right\}$ we can determine the corresponding ON basis $\left\{\Psi_{F a b}\right\}$ of positive energy solutions of (2.1),

$$
\begin{aligned}
\Psi_{F a b}(x)= & (2 \pi)^{-3 / 2} \iint_{-\infty}^{\infty} \int \exp (i k \cdot x) f_{F a b}(\mathrm{k}) d \mu(\mathrm{k}) \\
= & \pi^{-1} \sqrt{2} \exp [i(a+b)(\varphi-\pi / 2)]\left(\frac{(F-a) !(F-b) !}{(F+a) !(F+b) !}\right)^{1 / 2} \\
& \times \int_{0}^{\infty} \int_{0}^{\infty} \exp \left[\frac{1}{2}\left(i x_{0}-1\right)\left(\xi^{2}+\eta^{2}\right)\right. \\
& \left.-\frac{i}{2} x_{1}\left(\xi^{2}-\eta^{2}\right)\right](\xi \eta)^{a+b+1}
\end{aligned}
$$

$$
\times \sqrt{\times L_{F=a}^{(a+b)}\left(\xi^{2}\right) L_{F-b}^{(a+b)}\left(\eta^{2}\right) J_{a+b}(r \xi \eta) d \xi d \eta}
$$$$
x_{2}=r \sin \varphi, \quad x_{3}=r \cos \varphi \text {. }
$$

In terms of the coordinates

$$
\begin{aligned}
& x_{0}=\frac{\sin \psi}{y_{0}-\cos \psi}, \quad x_{1}=\frac{y_{1}}{y_{0}-\cos \psi}, \quad x_{2}=\frac{y_{2}}{y_{0}-\cos \psi}, \\
& x_{3}=\frac{y_{3}}{y_{0}-\cos \psi}, \quad y_{0}=\cos \alpha \cos \sigma, \quad y_{1}=\cos \alpha \sin \sigma, \\
& y_{2}=\sin \alpha \sin \varphi, \quad y_{3}=\sin \alpha \cos \varphi,
\end{aligned}
$$

we have

$$
\begin{aligned}
\Psi_{F a b}(x)= & (\cos \alpha \cos \sigma-\cos \psi) \exp [i(m \varphi+\rho \sigma-\{2 F+1) \Psi)] \\
& \times(2 \pi)^{-1 / 2}(-1)^{F-a+1}\left(\frac{(F+a) !(F+b) !}{\pi(F-a) !(F-b) !}\right)^{1 / 2}(\sin \alpha)^{a+b} \\
& \times \frac{(\cos \alpha)^{2 F-a-b}}{\Gamma(a+b+1)}{ }_{2} F_{1}\left(\begin{array}{l}
b-F, a-F \\
a+b+1
\end{array} \mid-\tan ^{2} \alpha\right)
\end{aligned}
$$

Indeed, direct computation shows

$$
\begin{gathered}
\Gamma_{56}=-\partial_{\phi}+\sin \psi(\cos \alpha \cos \sigma-\cos \psi)^{-1}, \quad \Gamma_{43}=\partial_{\varphi}, \\
\Gamma_{12}=\partial_{\sigma}+\cos \alpha \sin \sigma(\cos \alpha \cos \sigma-\cos \psi)^{-1}
\end{gathered}
$$

on the solution space of (2.1). Hence

$\Psi_{F a b}(x)=(\cos \alpha \cos \sigma-\cos \psi) \exp \{i[m \varphi+\rho \sigma-(2 F+1) \psi]\} g(\alpha)$ and substitution into (2.1) yields $R$-separation of variables. It follows from this that $g(\alpha)$ must be a multiple of $(\sin \alpha)^{a+b}(\cos \alpha)^{2 F-a-b}{ }_{2} F_{1}\left(b-F, a-F ; a+b+1 ;-\tan ^{2} \alpha\right)$. The constant is determined by explicitly computing (4.6) for convenient values of the variables.

There is another model of this irreducible representation which is very convenient for computations involving eigenfunctions of $\Gamma_{56}, \Gamma_{43}$, and $\Gamma_{12}$. The representation space $\mathcal{J}$ consists of functions $h$ of three complex 
variables $u, \imath, w$ such that $h(-u,-v,-w)=h(u, v, w)$. More precisely, $f$ is the complex Hilbert space with ON basis

$$
\begin{aligned}
& l_{F a b}=\left[\frac{(F+b) !(F-b) !}{(F+a) !(F-a) !}\right]^{1 / 2} F+a_{v} F+b_{w} 2 F-a-b, \\
& a, b=F, F-1, \ldots,-F, \quad 2 F=0,1, \cdots .
\end{aligned}
$$

The operators

$$
\begin{aligned}
\Gamma_{56}= & i\left(u \partial_{u}+v \partial_{v}+w \partial_{w}+1\right), \quad \Gamma_{43}=\frac{i}{2}\left(u \partial_{u}+v \partial_{v}-w \partial_{w}\right), \\
\Gamma_{12}= & i\left(u \partial_{u}-v \partial_{v}\right), \\
2 \Gamma_{16}= & i v\left(v \partial_{v}+1\right)-\frac{v^{-1} w^{-1}}{2}\left(-u \partial_{u}+v \partial_{v}+w \partial_{w}\right) \\
& +\frac{u w}{2}\left(u \partial_{u}-v \partial_{v}+w \partial_{w}+2\right)-u^{-1} w^{-1}\left(v \partial_{v}\right), \\
2 i P_{3}= & \left(u v-v w^{-1}\right)\left(v \partial_{v}+1\right)-\frac{1}{2}\left(\frac{u}{w}+\frac{v}{w^{2}}\right)\left(-u \partial_{u}+v \partial_{v}+w \partial_{w}\right) \\
& +\left(u v^{-1}-u^{-1} w\right)\left(u \partial_{u}\right)+\frac{1}{2}\left(w^{2}-v^{-1} w\right) \\
& \times\left(u \partial_{u}-v \partial_{v}+w \partial_{w}+2\right),
\end{aligned}
$$

acting on this basis satisfy relations (4.1), (4.3) and completely determine the action of so $(4,2)$. The three variable model appears to be the simplest in which to compute matrix elements of the SU(2,2) operators with respect to the $\left\{\Gamma_{56}, \Gamma_{43}, \Gamma_{12}\right\}$ eigenbasis. For some examples of matrix elements computed with this model see Ref. 18. (Indeed in this reference it is shown that one can choose another basis for the complexification of $\mathrm{so}(4,2)$ for which the differential operators take a much simpler form. The action of the Lie algebra on the basis $\left\{f_{F a b}\right\}$ corresponds exactly to the 12 known differential recurrence relations for the functions ${ }_{2} F_{1}$.)

We can see from (4.6) and (4.8) how one characterizes those solutions $\Psi$ of (2.1) such that $\Gamma_{56} \Psi=i \lambda \Psi$. It follows from these expressions that $\Psi=\left(y_{0}-\cos \psi\right) \Phi(y) \exp (-i \lambda \psi)$ where $y=\left(y_{1}, y_{1}, y_{2}, y_{3}\right)$ is an element of the sphere $S_{3}: y_{0}^{2}+y_{1}^{2}+y_{2}^{2}+y_{3}^{2}=1$. Moreover, Eq. (2.1) for $\Psi$ reduces to the eigenvalue equation

$$
\left(\Gamma_{12}^{2}+\Gamma_{13}^{2}+\Gamma_{14}^{2}+\Gamma_{23}^{2}+\Gamma_{24}^{2}+\Gamma_{34}^{2}\right) \Phi=\left(1-\lambda^{2}\right) \Phi .
$$

Here (4.11) is the eigenvalue equation for the LaplaceBeltrami operator on $S_{3}$. Indeed, the symmetry algebra of this equation is so(4) with basis $\left\{\Gamma_{i j}, 1 \leqslant i<j \leqslant 4\right\}$. The operators

$$
\begin{aligned}
& \Gamma_{12}=y_{0} \partial_{y_{1}}-y_{1} \partial_{y_{0}}, \quad \Gamma_{34}=y_{2} \partial_{y_{3}}-y_{3} \partial_{y_{2}}, \\
& \Gamma_{23}=y_{1} \partial_{y_{2}}-y_{2} \partial_{y_{1}}
\end{aligned}
$$

acting on $S_{3}$ generate this symmetry algebra.

Thus, the effect of diagonalizing $\Gamma_{56}$ is to reduce the separation of variables problem for (2.1) to the corresponding problem for (4.11). The latter equation was studied in Ref. 5 where it was shown that (4.11) separates in exactly six orthogonal coordinate systems, each corresponding to a commuting pair of symmetric second order symmetry operators from the enveloping algebra of so(4). Briefly, the list is

1] $\Gamma_{43}^{2}, \quad \Gamma_{12}^{2}$ (cylindrical)

2] $\Gamma_{12}^{2}+\Gamma_{13}^{2}+\Gamma_{23}^{2}, \quad \Gamma_{12}^{2}$ (spherical)
3] $\Gamma_{12}^{2}+\Gamma_{13}^{2}+\Gamma_{23}^{2}, \quad \Gamma_{23}^{2}+k^{2} \Gamma_{13}^{2}, \quad 0<k<1 \quad$ (spheroelliptic)

4) $\Gamma_{12}^{2}+\Gamma_{13}^{2}+\Gamma_{23}^{2}+a\left(\Gamma_{12}^{2}-\Gamma_{34}^{2}\right), \quad \Gamma_{12}^{2}, \quad-\infty<a<-1$

(elliptic cylindrical, Type I)

5] Same as 4] with $-1<a<0$ (elliptic cylindrical, Type II)

$$
\text { 6] } \begin{aligned}
& \Gamma_{23}^{2}-\Gamma_{14}^{2}+\left(\frac{a-b-1}{1-a-b}\right)\left(\Gamma_{13}^{2}-\Gamma_{24}^{2}\right)+\left(\frac{b-a-1}{1-a-b}\right) \\
& \times\left(\Gamma_{12}^{2}-\Gamma_{34}^{2}\right), \\
& 2 b(1-a)\left(\Gamma_{13}^{2}+\Gamma_{24}^{2}\right)+2 a(1-b)\left(\Gamma_{12}^{2}+\Gamma_{34}^{2}\right) \\
& +2 b(1-a)\left(\Gamma_{13}^{2}-\Gamma_{24}^{2}\right)+2 a(1-b)\left(\frac{a-b-1}{1-a-b}\right)\left(1_{12}^{2}-\Gamma_{34}^{2}\right) \\
& \text { (ellipsoidal). }
\end{aligned}
$$

The names of the separable coordinates are listed in parentheses. These systems are studied in detail in Ref. 5 and related to the hydrogen atom eigenvalue equation.

\section{DIAGONALIZATION OF $P_{0}, P_{3}{ }^{\prime}$, AND D}

Next we search for coordinate systems permitting separation of variables in (2.1) such that the corresponding basis functions $\Psi$ are eigenfunctions of $P_{0}: F_{0} \Psi$ $=i \omega \Psi$. In this case we can set $\Psi(x)=\exp \left(i \omega x_{0}\right) \Phi\left(x_{1}, x_{2}, x_{3}\right)$ where

$$
\left(\partial_{11}+\partial_{22}+\partial_{33}+\omega^{2}\right) \Phi=0 .
$$

It follows that the reduced equation for the eigenfunctions is the Helmholtz equation. The symmetry algebra for (5.1) is $\varepsilon(3)$, the Lie algebra of the Euclidean group in three-space. A basis for $\mathcal{E}(3)$ is $\left\{P_{1}, P_{2}, P_{3}, M_{21}, M_{13}, M_{32}\right\}$. It is well known ${ }^{2,19}$ that this equation separates in exactly 11 orthogonal coordinate systems, each system corresponding to a pair of commuting second order symmetric operators in the enveloping algebra of $\mathcal{E}(3)$. Briefly, the separable systems are

1] $P_{2}^{2}, \quad P_{3}^{2}$ (Cartesian),

2] $M_{21}^{2}, \quad P_{3}^{2}$ (cylindrical),

3] $\left\{M_{21}, P_{2}\right\}, \quad P_{3}^{2}$ (parabolic cylindrical),

4] $M_{21}^{2}+d^{2} P_{1}^{2}, \quad P_{3}^{2}, \quad d=0$ (elliptic cylindrical),

5] $M_{21}^{2}+M_{13}^{2}+M_{32}^{2}, \quad M_{21}^{2}$ (spherical),

6] $M_{21}^{2}+M_{13}^{2}+M_{32}^{2}-a^{2}\left(P_{1}^{2}+P_{2}^{2}\right), \quad M_{21}^{2}, \quad a>0$

(prolate spheroidal),

7] $M_{21}^{2}+M_{13}^{2}+M_{32}^{2}+a^{2}\left(P_{1}^{2}+H_{2}^{2}\right), \quad M_{21}^{2}, \quad a=0$

(oblate spheroidal),

8] $\left\{M_{32}, P_{2}\right\}-\left\{M_{13}, P_{1}\right\}, M_{21}^{2}$ (parabolic),

9] $M_{21}^{2}-c^{2} P_{3}^{2}+c\left(\left\{M_{13}, P_{1}\right\}+\left\{M_{32}, P_{2}\right\}\right)$,

$c\left(P_{2}^{2}-P_{1}^{2}\right)+\left\{M_{13}, P_{1}\right\}-\left\{M_{32}, P_{2}\right\}$ (paraboloidal),

10] $P_{1}^{2}+a P_{2}^{2}+(a+1) P_{3}^{2}+M_{21}^{2}+M_{13}^{2}+M_{32}^{2}$,

$M_{13}^{2}+a\left(M_{32}^{2}+P_{3}^{2}\right), \quad a>1 \quad$ (ellipsoidal),

11] $M_{21}^{2}+M_{13}^{2}+M_{32}^{2}, \quad M_{32}^{2}+b M_{13}^{2}, \quad 1>b>0$

(conical). Here $\{A, B\}=A B+B A$. 
On $H$ the condition $P_{0} f=i \omega f$ implies $f(\mathbf{k})=\delta\left(k_{0}-\omega\right) g_{\omega}(\hat{\mathbf{k}})$ where $\omega>0$ and $\mathbf{k}=\left(k_{1}, k_{2}, k_{3}\right)$ ranges over the unit sphere $S_{2}: \hat{\mathbf{k}} \cdot \hat{\mathbf{k}}=1, \mathbf{k}=\omega \hat{\mathbf{k}}$. To determine the functions $g_{\omega}$ one uses the Hilbert space $L_{2}\left(S_{2}\right)$ of square integrable functions on $S_{2}$ [with measure $d \Omega(\hat{\mathbf{k}})=d \hat{k}_{1} \cdot d \hat{k}_{2} / \hat{k}_{3}$ ] on which $\varepsilon(3)$ acts via

$$
\begin{aligned}
& P_{j}=-i \omega \hat{k}_{j}, \quad M_{j l}=\hat{k}_{j} \partial_{\hat{k}_{l}}-\hat{k}_{l} \partial_{\hat{k}_{j}}, \\
& 1 \leqslant j, l \leqslant 3, \quad j \neq l .
\end{aligned}
$$

These operators determine a unitary irreducible representation of $\mathrm{E}(3)$ on $L_{2}\left(S_{2}\right){ }^{20}$ Once the eigenfunctions $g_{\alpha \beta}(\hat{k})$ of the operator pairs 1]-11] have been determined, the corresponding separable solutions $\Psi_{\omega \alpha \beta}(x)$ of $(2.1)$ can be obtained from the integral transform

$$
\Psi_{\omega \alpha \beta}(x)=\frac{\omega \exp \left(i \omega x_{0}\right)}{(2 \pi)^{3 / 2}} \int_{S_{2}} \exp (i \omega \mathbf{x} \cdot \hat{\mathbf{k}}) g_{\alpha \beta}(\hat{\mathbf{k}}) d \Omega(\hat{\mathbf{k}}) .
$$

All the eigenfunctions $g_{\alpha \beta}$ and integrals (5.3) have been computed in Ref. 2 .

Now we study coordinate systems permitting variable separation in (2.1) such that the basis functions $\Psi$ are eigenfunctions of $P_{3}: P_{3} \Psi=-i \lambda \Psi$. Here we can set $\Psi(x)$ $=\exp \left(-i \lambda x_{3}\right) \Phi\left(x_{0}, x_{1}, x_{2}\right)$, where

$$
\left(\partial_{00}-\partial_{11}-\partial_{22}+\lambda^{2}\right) \Phi=0 \text {. }
$$

The symmetry algebra of the Klein-Gordon equation (5.4) is $\mathcal{E}(2,1)$ with basis $\left\{P_{0}, P_{1}, P_{2}, M_{21}, M_{01}, M_{02}\right\}$. Furthermore, the pseudo-Euclidean (or Poincarê) group $\mathrm{E}(2,1)$ is the symmetry group of (5.4). In Ref. 3 it is shown in detail that variables separate in (5.4) for 53 orthogonal coordinate systems, each system characterized by a pair of commuting second-order symmetric operators in the enveloping algebra of $\varepsilon(2,1)$. [Of course the coordinates 1]-4] for (5.1) are counted again in the list of 53 systems for (5.4).]

On $H$ the requirement $P_{3} f=-i \lambda f$ implies $f(\mathbf{k})=\delta\left(k_{3}\right.$ $-\lambda) g\left(k_{1}, k_{2}\right)$ where $-\infty<\lambda<\infty$. The search for eigenfunctions reduces to a study of the Hilbert space $L_{2}(H)$ of square integrable functions with respect to the measure $d \zeta=d k_{1} d k_{2} / k_{0}$, where $k_{0}=\left(k_{1}^{2}+k_{2}^{2}+\lambda^{2}\right)^{1 / 2}$. The inner product is

$$
\left(h, h^{\prime}\right)=\iint_{-\infty}^{\infty} h\left(k_{1}, k_{2}\right) \bar{h}^{\prime}\left(k_{1}, k_{2}\right) d \zeta, \quad h, h^{\prime} \in L_{2}(H),
$$

and the action of $\mathcal{E}(2,1)$ on $L_{2}(H)$ is given by

$$
\begin{aligned}
& P_{0}=i k_{0}, \quad P_{1}=-i k_{1}, \quad P_{2}=-i k_{2}, \\
& M_{21}=k_{2} \partial_{k_{1}}-k_{1} \partial_{k_{2}}, \quad M_{01}=k_{0} \partial_{k_{1}}, \quad M_{02}=k_{0} \partial_{k_{2}} .
\end{aligned}
$$

As is well known, ${ }^{1,20}$ these operators define a unitary irreducible representation of $\mathrm{E}(2,1)$ on $L_{2}(H)$. Once the eigenfunctions $g_{\alpha \beta}$ corresponding to each of the 53 separable systems have been determined, the associated separable solutions of (2.1) follow from

$$
\begin{aligned}
\Psi_{\lambda \alpha \beta}(x)= & \frac{\exp \left(-i \lambda x_{3}\right)}{(2 \pi)^{3 / 2}} \iint_{-\infty}^{\infty} \exp \left[i \left(k_{0} x_{0}-k_{1} x_{1}\right.\right. \\
& -k_{2} x_{2} \lg _{\alpha \beta}\left(k_{1}, k_{2}\right) d \zeta .
\end{aligned}
$$

A detailed study of the basis functions $g_{\alpha \beta}$ and the integrals (5.7) has not yet been undertaken.
Now we search for separable coordinate systems for (2.1) such that the corresponding basis functions $\Psi$ are eigenfunctions of $D: D \Psi=-i \nu \Psi$. Then we have $\Psi(x)$ $=\rho^{i \nu-1} \Phi\left(s_{0}, s_{1}, s_{2}, s_{3}\right)$, where $x_{\alpha}=\rho s_{\alpha}, \rho \geqslant 0, s \cdot s=\epsilon$, and $\epsilon=+1,-1$, or 0 depending on whether $x \cdot x>0$, $<0$, or $=0$. From Eq. (2.24ii) we see that the reduced equation for $\Phi$ is

$$
\left(M_{21}^{2}+M_{13}^{2}+M_{32}^{2}-M_{01}^{2}-M_{02}^{2}-M_{03}^{2}\right) \Phi(s)=\left(\nu^{2}+1\right) \Phi(s) .
$$

The operator $D$ commutes with the subalgebra so $(3,1)$ with basis $\left\{M_{21}, M_{13}, M_{32}, M_{01}, M_{02}, M_{03}\right\}$ and, in fact, so $(3,1)$ is the symmetry algebra of $(5.8)$.

As discussed in Ref. 6, (5.8) separates in 34 orthogonal coordinate systems for the case $\epsilon=+1$, each system characterized by a pair of commuting second-order symmetric operators in the enveloping algebra of so $(3,1)$. Some results for the case $\epsilon=-1$ are also presented in Ref. 6 .

On $H$ the requirement $D f=-i \nu f$ implies $f(\mathbf{k})=k_{0}^{i \nu-1} h_{\nu}(\hat{\mathrm{k}})$, $-\infty<\nu<\infty$, where $\mathbf{k}=k_{0} \hat{\mathbf{k}}$ and $\hat{\mathbf{k}}$ ranges over the unit sphere $S_{3}$. The eigenfunction problem reduces to a study of the Hilbert space $L_{2}\left(S_{3}\right)$ on which $\operatorname{so}(3,1)$ acts via

$$
\begin{aligned}
& M_{21}=\hat{k}_{2} \partial_{\hat{k}_{1}}-\hat{k}_{1} \partial_{\hat{k}_{2}}, \quad M_{13}=-\hat{k}_{3} \partial_{\hat{k}_{1}}, \quad M_{32}=\hat{k}_{3} \partial_{\hat{k}_{2}}, \\
& M_{01}=-(1+i \nu) \hat{k}_{1}+\left(1-\hat{k}_{1}^{2}\right) \partial_{\hat{k}_{1}}-\hat{k}_{1} \hat{k}_{2} \partial_{\hat{k}_{2}}, \\
& M_{02}=-(1+i \nu) \hat{k}_{2}-\hat{k}_{1} \hat{k}_{2} \partial_{\hat{k}_{1}}+\left(1-\hat{k}_{2}^{2}\right) \partial_{\hat{k}_{2}}, \\
& M_{03}=-(1+i \nu) \hat{k}_{3}-\hat{k}_{1} \hat{k}_{3} \partial_{k_{1}}-\hat{k}_{1} \hat{k}_{3} \partial_{\hat{k}_{2}},
\end{aligned}
$$

where we have chosen $\hat{k}_{1}, \hat{k}_{2}$ as the independent variables. These operators determine an irreducible unitary global representation of $\operatorname{SO}(3,1)$ which belongs to the principal series. Once the eigenfunctions $h_{\nu \alpha \beta}(\hat{\mathbf{k}})$ for each of the 34 separable systems have been determined the corresponding separable solutions $\Psi_{\nu \alpha \beta}$ of $(2.1)$ can be obtained from

$$
\begin{aligned}
\Psi_{\nu \alpha \beta}(x)= & \frac{\rho^{i \nu-1}}{(2 \pi)^{3 / 2}} \Gamma(1-i \nu) \\
& \times \iint_{s_{3}} \exp [ \pm \pi(i+\nu) / 2]\left|1-\hat{k}_{1} s_{1}-\hat{k}_{2} s_{2}-\hat{k}_{3} s_{3}\right|^{i \nu-1} \\
& \times h(\hat{\mathbf{k}}) d \Omega(\hat{\mathbf{k}})
\end{aligned}
$$

where the plus sign occurs when $1-\hat{\mathbf{k}} \cdot \mathbf{s}>0$ and the minus sign occurs when $1-\hat{\mathbf{k}} \cdot \mathbf{s}<0$. For the case $\epsilon=+1$, $x_{0}>0$, these integrals are evaluated in Ref. 6. A number of cases for $\epsilon=-1$ are also computed.

\section{THE SCHRÖDINGER EQUATION}

Now we consider the separable coordinate systems for (2.1) such that the basis functions $\Psi$ are eigenfunctions of $P_{0}+P_{1}:\left(P_{0}+P_{1}\right) \Psi=i \beta \Psi$. Setting $\Psi(x)=\exp (i s \beta)$ $\times \Phi\left(t, x_{2}, x_{3}\right)$, where $2 s=x_{0}+x_{1}, 2 t=x_{1}-x_{0}$, we find that the reduced equation satisfied by $\Phi$ is the free particle Schrödinger equation

$$
\left(i \beta \partial_{t}+\partial_{22}+\partial_{33}\right) \Phi\left(t, x_{2}, x_{3}\right)=0,
$$

which admits as symmetries the operators

$$
\begin{aligned}
& P_{1}=P_{2}, \quad P_{2}=P_{3}, \quad \mathcal{E}=P_{0}+P_{1}, \quad K_{-2}=P_{1}-P_{0}, \\
& K_{2}=-\frac{1}{2}\left(K_{0}+K_{1}\right), \quad M=-M_{32}, \quad B_{1}=\frac{1}{2}\left(M_{02}+M_{21}\right), \\
& B_{2}=\frac{1}{2}\left(M_{03}-M_{13}\right), \quad D=-\left(D+M_{01}\right) .
\end{aligned}
$$


All these operators commute with $\varepsilon=P_{0}+P_{1}$ and they form a basis for the nine-dimensional symmetry algebra $G_{2}$ of (6.1). This algebra is discussed in Refs. 7 and 21 . In Ref. 7 it is shown that (6.1) admits $R$-separable solutions in 17 (nonorthogonal) coordinate systems. Each system is characterized by a commuting pair of symmetry operators from the enveloping algebra of $\hat{y}_{2}$, one operator first-order and one second-order.

On $H$ the requirement $\left(P_{0}+P_{1}\right) f=i \beta f$ implies $f(\mathbf{k})$ $=u \delta(u-\beta) l_{\beta}(v, w)$, where $\beta>0, u=k_{0}-k_{1}, v=k_{2}, w=k_{3}$. The search for eigenfunctions $l_{\beta}$ reduces to a study of the Hilbert space $L_{2}\left(R^{2}\right)$ on which the Schrödinger algebra acts via

$$
\begin{aligned}
& P_{1}=-i v, \quad P_{2}=-i w, \quad \varepsilon=i \beta, \quad K_{-2}=\frac{-i}{\beta}\left(v^{2}+w^{2}\right), \\
& K_{2}=\frac{-i \beta}{4}\left(\partial_{v v}+\partial_{w w}\right), \quad M=v \partial_{w}-w \partial_{v}, \\
& B_{1}=\frac{1}{2} \beta \partial_{v}, \quad B_{2}=\frac{1}{2} \beta \partial_{w}, \quad D=-\left(1+v \partial_{v}+w \partial_{w}\right) .
\end{aligned}
$$

It is known ${ }^{7,22}$ that these operators induce a unitary irreducible representation of the Schrödinger group $G_{2}$ on $L_{2}\left(R^{2}\right)$. Once the eigenfunctions $l_{B \alpha \rho}(v, w)$ corresponding to each separable system have been determined, the corresponding separable solutions $\Psi_{\beta \alpha \rho}(x)$ of $(2.1)$ follow from

$$
\begin{aligned}
\Psi_{\beta \alpha \rho}(x)= & \frac{\exp (i \beta s)}{(2 \pi)^{3 / 2}} \iint_{-\infty}^{\infty} \exp \left[\frac{-i t}{\beta}\left(v^{2}+w^{2}\right)\right. \\
& \left.-i\left(x_{2} v+x_{3} w\right)\right] l_{\beta \alpha \rho}(v, w) d v d w .
\end{aligned}
$$

Using the $u, v, w$ coordinates we can now compute the operator $\exp \left[a\left(K_{0}+K_{1}\right)\right]$ in $H$. Indeed the well-known expression

$$
\begin{aligned}
& \exp \left[i t\left(\partial_{x x}+\partial_{y y}\right)\right] f(x, y) \\
&= \text { l。 i.m. } \frac{1}{4 \pi t} \iint_{-\infty}^{\infty} \exp \left\{-\frac{1}{4 i t}\left[\left(x-s_{1}\right)^{2}\right.\right. \\
&\left.\left.+\left(y-s_{2}\right)^{2}\right]\right\} f\left(s_{1}, s_{2}\right) d s_{1} d s_{2},
\end{aligned}
$$

for time translation of solutions of the free-particle Schrödinger equation, e.g.,${ }^{7}$ together with expressions (6.2) and (6.3) for $K_{-2}$ leads to

$$
\begin{aligned}
\exp \left[a\left(K_{0}+K_{1}\right)\right] f(\mathrm{k}) & \\
= & \frac{1}{4 \pi i a} \iint_{-\infty}^{\infty} \exp \left\{\frac{1}{-4 a i\left(k_{0}-k_{1}\right)}\left[\left(k_{2}-s_{2}\right)^{2}+\left(k_{3}-s_{3}\right)^{2}\right]\right\} \\
& \times f\left(\frac{s_{2}^{2}+s_{3}^{2}-\left(k_{0}-k_{1}\right)^{2}}{2\left(k_{0}-k_{1}\right)}, s_{2}, s_{3}\right) d s_{2} d s_{3}, \quad f \in H
\end{aligned}
$$

\section{THE GENERALIZED EPD EQUATION}

We next look for solutions $\Psi$ of (2.1) such that $\Gamma_{43} \Psi$ $=i m \Psi$. Then $\Psi(x)=\exp (i m \varphi) \Phi\left(x_{0}, x_{1}, r\right)$ where

$$
x_{3}=r \cos \varphi, \quad x_{2}=r \sin \varphi
$$

and $\Phi$ satisfies the reduced equation

$$
\left(\partial_{0 u}-\partial_{r r}-\frac{1}{\gamma^{2}} \partial_{r}+\frac{m^{2}}{\gamma^{2}}-\partial_{11}\right) \Phi=0 .
$$

If $\Phi$ is independent of $x_{1}$ then (7.1) reduces to the EulerPoisson-Darboux (EPD) equation. Expression (7.1) can be written in the operator form (2.24iv),

$\left(\Gamma_{12}^{2}+\Gamma_{56}^{2}-\Gamma_{15}^{2}-\Gamma_{16}^{2}-\Gamma_{26}^{2}-\Gamma_{25}^{2}\right) \Phi=\left(\Gamma_{43}^{2}+1\right) \Phi=\left(1-m^{2}\right) \Phi$.

The symmetry algebra of $(7.1)$ is $s o(2,2)$ with basis $\left\{\Gamma_{12}, \Gamma_{56}, \Gamma_{15}, \Gamma_{16}, \Gamma_{26}, \Gamma_{25}\right\}$ or alternate basis $\left\{P_{0}, K_{0}, P_{1}\right.$, $\left.K_{1}, D, M_{01}\right\}$. Separable coordinate systems for this interesting equation will be classified in a later publication.

On $H$ the requirement $\Gamma_{43} f=i m f$ implies $f(\mathrm{k})=\exp (i m \theta)$ $\times j(l, k)$ where $m=0, \pm 1, \pm 2, \cdots, l \geqslant 0, k_{3}=l \cos \theta, k_{2}$ $=l \sin \theta, k_{1}=k$. The eigenfunction problem reduces to a study of the Hilbert space $L_{2}$ of functions $j(l, k)$ Lebesgue square integrable with respect to the measure $d \rho(l, k)$ $=l\left(l^{2}+k^{2}\right)^{-1 / 2} d l d k$. The inner product is

$$
\left(j_{1}, j_{2}\right)=\int_{0}^{\infty} \int_{-\infty}^{\infty} j_{1} \bar{j}_{2} d \rho(l, k), \quad j_{1}, j_{2} \in L_{2} .
$$

The symmetry algebra $\operatorname{so}(2,2)$ acts on $L_{2}$ via

$$
\begin{aligned}
& \Gamma_{12}=\frac{i k}{2}\left(\partial_{k k}-\partial_{l l}-l^{-1} \partial_{l}+m^{2} l^{-2}-1\right)+i l \partial_{l k}-\partial_{k}, \\
& \Gamma_{56}=\frac{i}{2}\left(k^{2}+l^{2}\right)^{1 / 2}\left(-\partial_{l l}-l^{-1} \partial_{l}+m^{2} l^{-2}-\partial_{k k}+1\right), \\
& \Gamma_{15}=\frac{i}{2}\left(k^{2}+l^{2}\right)^{1 / 2}\left(\partial_{l l}+l^{-1} \partial_{l}-m^{2} l^{-2}+\partial_{k k}+1\right), \\
& \Gamma_{16}=1+l \partial_{l}+k \partial_{k}, \\
& \Gamma_{26}=\frac{i k}{2}\left(-\partial_{k k}+\partial_{l l}+l^{-1} \partial_{l}-m^{2} l^{-2}-1\right)-i l \partial_{l k}-i \partial_{k}, \\
& \Gamma_{25}=\left(k^{2}+l^{2}\right)^{1 / 2} \partial_{k} .
\end{aligned}
$$

A third basis of $\operatorname{so}(2,2)$ for which the structure of the Lie algebra becomes more transparent is

$$
\begin{array}{lll}
A_{1}=\Gamma_{56}+\Gamma_{12}, & A_{2}=\Gamma_{52}+\Gamma_{16}, & A_{3}=\Gamma_{26}+\Gamma_{15}, \\
B_{1}=\Gamma_{56}-\Gamma_{12}, & B_{2}=\Gamma_{52}-\Gamma_{16}, & B_{3}=\Gamma_{26}-\Gamma_{15},
\end{array}
$$

which commutation relations

$$
\begin{aligned}
& {\left[A_{1}, A_{2}\right]=-2 A_{3}, \quad\left[A_{2}, A_{3}\right]=2 A_{1}, \quad\left[A_{1}, A_{3}\right]=2 A_{2},} \\
& {\left[B_{1}, B_{2}\right]=-2 B_{3}, \quad\left[B_{2}, B_{3}\right]=2 B_{1}, \quad\left[B_{1}, B_{3}\right]=2 B_{2} \text {, }} \\
& {\left[A_{i}, B_{j}\right]=0 \text {. }}
\end{aligned}
$$

With respect to this basis the isomorphism so $(2,2)$ $\approx \mathrm{sl}(2) \times \mathrm{sl}(2)$ is obvious. Moreover, it follows from (4.1) and (4.2) that in the eigenspace $H_{m}$ of $H$ corresponding to the eigenvalue $m$ of $-i \Gamma_{43}$ there is an ON basis $\left\{f_{\alpha \beta}\right\}$ such that

$$
\begin{aligned}
& A_{1} f_{\alpha \beta}=i(|m|+2 \alpha+1) f_{\alpha \beta}, \quad B_{1} f_{\alpha \beta}=i(|m|+2 \beta+1) f_{\alpha \beta}: \\
& \alpha, \beta=0,1,2, \cdots .
\end{aligned}
$$

Also $A_{1}^{2}-A_{2}^{2}-A_{3}^{2}=B_{1}^{2}-B_{2}^{2}-B_{3}^{2}=1-m^{2}$ on $H_{m}$. It follows that this action of $\operatorname{sl}(2) \times \operatorname{sl}(2)$ on $H_{m}$ is irreducible and extends to a unitary irreducible representation $D_{(|m|-1) / 2}^{-}$ $\otimes D_{(|m|-1) / 2}^{-}$of $\mathrm{SL}(2, R) \times \mathrm{SL}(2, R)$ on $H_{m}$. Here $D_{k}^{-}$is a representation of $\mathrm{SL}(2, R)$ belonging to the negative discrete series.

Once the eigenbasis $\left\{g_{\xi \eta}\right\}$ in $H_{m}$ corresponding to a separable system for (7.1) has been constructed, the associated separable solutions of (7.1) can be obtained from the transform 


$$
\begin{aligned}
\Psi_{m \xi \eta}(x)= & \frac{\exp [i m(\varphi-\pi / 2)]}{(2 \pi)^{1 / 2}} \int_{0}^{\infty} l d l J_{m}(l \gamma) \int_{-\infty}^{\infty} d k \\
& \cdot \exp \left[i\left(x_{0}\left(k^{2}+l^{2}\right)^{1 / 2}-x_{1} k\right)\right] j(l, k) /\left(k^{2}+l^{2}\right)^{1 / 2}
\end{aligned}
$$

\section{DIAGONALIZATION OF $\Gamma_{12}+\Gamma_{34}-\Gamma_{56}$}

Finally we study the separable solutions of (2.1) for which the basis functions $\Psi$ are eigenfunctions of $L$ $=\Gamma_{12}+\Gamma_{34}-\Gamma_{56}: L \Psi=-i(2 \kappa+1) \Psi$. By a tedious computation one can verify that (2.1) is equivalent to the equation

$$
\left(\frac{1}{3} A_{0}^{2}+A_{1}^{2}+A_{2}^{2}+A_{3}^{2}-B_{1}^{2}-B_{2}^{2}-C_{1}^{2}-C_{2}^{2}\right) \Psi=\left(\frac{1}{3} L^{2}+3\right) \Psi,
$$

where

$$
\begin{aligned}
& A_{0}=\Gamma_{34}+\Gamma_{12}+2 \Gamma_{56}, \quad A_{1}=\Gamma_{13}+\Gamma_{24}, \quad A_{2}=\Gamma_{12}-\Gamma_{34}, \\
& A_{3}=\Gamma_{14}-\Gamma_{23}, \quad B_{1}=\Gamma_{25}-\Gamma_{15}, \quad B_{2}=\Gamma_{45}-\Gamma_{36}, \\
& C_{1}=\Gamma_{15}+\Gamma_{26}, \quad C_{2}=\Gamma_{35}+\Gamma_{46} .
\end{aligned}
$$

Thus, the reduced equation is

$$
\begin{aligned}
\left(\frac{1}{3} A_{0}^{2}+A_{1}^{2}+A_{2}^{2}+A_{3}^{2}-B_{1}^{2}-B_{2}^{2}-C_{1}^{2}-C_{2}^{2}\right) \Psi & =\left(-\mu^{2} / 3+3\right) \Psi, \\
\mu & =2 \kappa+1 。
\end{aligned}
$$

The operators (8.2) satisfy the commutation relations for $\operatorname{su}(2,1)$ and the expression on the left-hand side of (8.3) is the Casimir operator for $\operatorname{su}(2,1)$.

The usual model for su(2,1) is the space of $3 \times 3$ complex matrices $A$ such that

$$
\not^{t} G^{2,1}+G^{2,1} A=0
$$

where $\mathrm{e}^{23}$

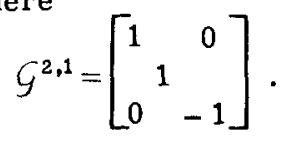

This real Lie algebra is eight-dimensional with basis

$$
\begin{aligned}
& A_{0}=\left[\begin{array}{lll}
i & 0 & 0 \\
0 & i & 0 \\
0 & 0 & -2 i
\end{array}\right], A_{1}=\left[\begin{array}{lll}
0 & 1 & 0 \\
-1 & 0 & 0 \\
0 & 0 & 0
\end{array}\right], A_{2}=\left[\begin{array}{lll}
i & 0 & 0 \\
0 & -i & 0 \\
0 & 0 & 0
\end{array}\right], \\
& A_{3}=\left[\begin{array}{lll}
0 & i & 0 \\
i & 0 & 0 \\
0 & 0 & 0
\end{array}\right], B_{1}=\left[\begin{array}{lll}
0 & 0 & 1 \\
0 & 0 & 0 \\
1 & 0 & 0
\end{array}\right], B_{2}=\left[\begin{array}{lll}
0 & 0 & 0 \\
0 & 0 & 1 \\
0 & 1 & 0
\end{array}\right],
\end{aligned}
$$

$$
C_{1}=\left[\begin{array}{ccc}
0 & 0 & i \\
0 & 0 & 0 \\
-i & 0 & 0
\end{array}\right], C_{2}=\left[\begin{array}{ccc}
0 & 0 & 0 \\
0 & 0 & i \\
0 & -i & 0
\end{array}\right],
$$

and the basis elements satisfy the same commutation relations as the corresponding operators (8.2). The symmetry algebra of $\mathrm{Eq} .(8.3)$ is also $\operatorname{su}(2,1)$.

It follows from (4.1) and (4.2) that the possible values of $\kappa$ are $0,1,2, \cdots$ and for fixed $\kappa$, the solution space of (8. 3) has an oN basis $\left\{\Psi_{l, s}: l=0,1,2, \cdots, s=0,1, \ldots\right.$, $\kappa+l\}$ such that

$$
\begin{aligned}
& L \Psi_{l s}=-i(2 \kappa+1) \Psi_{l s}, \quad A_{0} \Psi_{l s}=i(\kappa+3 l+2) \Psi_{l s}, \\
& A_{2} \Psi_{l s}=i(2 s-\kappa-l) \Psi_{l s} .
\end{aligned}
$$

The solution space of (8.3) transforms irreducibly under this action of $\mathrm{su}(2,1)$ and the Lie algebra representation lifts to a global unitary irreducible representation of $\mathrm{SU}(2,1)$, see Ref. 24.

The problem of separation of variables for $(8,3)$ is far from settled. The variables in (8.3) are intertwined in an extremely complicated manner and the standard techniques for separating variables in the wave equation, e.g., Ref. 25, yield no nontrivial separable systems for this case. However, it follows from standard Lie theory, Ref. 26 , p. 49 , that every pair of commuting operators in $\mathrm{su}(2,1)$ leads to a separable coordinate system. It is not yet known whether there exist separable systems corresponding to second-order operators in the enveloping algebra of $\mathrm{su}(2,1)$.

\section{CONCLUDING REMARKS}

For completeness we classify the orbits in so(4,2) under the adjoint action of $\mathrm{SO}(4,2)$. This classification has been given by Zassenhaus ${ }^{27}$ and later by many others but we present the results here in an explicit form adapted to our notation. (This orbit analysis is useful because we know that coordinate systems whose defining operators can be mapped into one another under an action of the adjoint group are equivalent.) We list the possible eigenvalues of a $6 \times 6$ matrix $A \in \mathrm{so}(4,2)$ such that $\Gamma$ $=T A T^{-1}$ for some $T \in \mathrm{SO}(4,2)$, i.e., we list an element on each $\mathrm{SO}(4,2)$ orbit. It is easy to show that if $\lambda \neq 0$ is an eigenvalue then so are $-\lambda$ and $\bar{\lambda}$. We use the notation $\lambda(n), n=2, \ldots, 5$, to signify that $\lambda$ corresponds to a generalized eigenvector $x$ of rank $n$, i.e., $n$ is the smallest integer $m$ such that $(A-\lambda E)^{m} x=0$ where $E$ is the $6 \times 6$ identity matrix.
Possible eigenvalues

$$
\begin{aligned}
& \text { 1. } \pm \alpha \pm i \beta, \pm i \gamma, \\
& \alpha, \beta \neq 0 \\
& \text { 2. } \pm i \alpha, \pm \beta, \pm \gamma, \\
& \beta^{2}+\gamma^{2}>0 \\
& \text { 3. } \pm i \alpha, \pm i \beta, \quad \pm i \gamma, \\
& \alpha, \beta, \gamma \neq 0
\end{aligned}
$$

3a. $\pm i \alpha, \pm i \beta, \quad 0, \quad 0$, $\alpha, \beta \neq 0$

3b. $\pm i \alpha, \quad 0, \quad 0, \quad 0, \quad 0$
Canonical form $T$

$$
\begin{aligned}
& \gamma \Gamma_{12}+\beta\left(\Gamma_{34}+\Gamma_{65}\right)+\alpha\left(\Gamma_{35}+\Gamma_{46}\right) \\
& \alpha \Gamma_{12}+\beta \Gamma_{35}+\gamma \Gamma_{46} \\
& \alpha \Gamma_{12}+\beta \Gamma_{34}+\gamma \Gamma_{56} \\
& \alpha \Gamma_{12}+\beta \Gamma_{34} \text { or } \alpha \Gamma_{12}+\beta \Gamma_{56} \\
& \alpha \Gamma_{12} \text { or } \alpha \Gamma_{56}
\end{aligned}
$$



4. $\alpha(2),-\alpha(2), \pm i \beta$
$\alpha\left(\Gamma_{35}+\Gamma_{46}\right)+\beta \Gamma_{12}+\frac{1}{2}\left(\Gamma_{34}+\Gamma_{36}+\Gamma_{45}+\Gamma_{65}\right)$
$\alpha \neq 0$
5. $i \alpha(3),-i \alpha(3)$
6. $i \alpha(2),-i \alpha(2), \pm i \beta$
$\alpha\left(\Gamma_{12}+\Gamma_{34}+\Gamma_{65}\right)+\frac{1}{2}\left(\Gamma_{13}+\Gamma_{24}+\Gamma_{15}+\Gamma_{26}\right)$
7. $\pm \alpha, \quad 0(3), 0$, $\alpha \neq 0$
8. $\pm i \alpha, \quad 0(3), \quad 0$
$\alpha\left(\Gamma_{34}+\Gamma_{65}\right)+\beta \Gamma_{12}+\frac{1}{2}\left(\Gamma_{34}+\Gamma_{56}+\Gamma_{36}+\Gamma_{54}\right)$
$\alpha \Gamma_{46}+\frac{1}{\sqrt{2}}\left(\Gamma_{21}+\Gamma_{25}\right)$
$\alpha \Gamma_{12}+\frac{1}{\sqrt{2}}\left(\Gamma_{35}+\Gamma_{65}\right)$ or $\alpha \Gamma_{12}+\frac{1}{\sqrt{2}}\left(\Gamma_{43}+\Gamma_{45}\right)$
9. $0(5), \quad 0$$$
\frac{1}{2}\left(\Gamma_{43}+\Gamma_{36}+\Gamma_{45}+\Gamma_{56}\right)+\frac{1}{\sqrt{2}}\left(\Gamma_{24}+\Gamma_{26}\right)
$$

From these results we can see why many operators $\Gamma \in s o(4,2)$ do not directly correspond to a semisubgroup coordinate system. For example, it is easy to check that an element of so(4,2) which commutes with $\Gamma$ (case 1], $\alpha, \beta, \gamma \neq 0$ ) also commutes with each of the (commuting) operators $\Gamma_{12}, \Gamma_{34}+\Gamma_{65}$, and $\Gamma_{35}+\Gamma_{46}$. The coordinate system associated with these operators is equivalent to a separable system for Eq. (7.1). By interpreting the remaining cases in a similar fashion one can show that each case is in fact associated with at least one semisubgroup coordinate system.

${ }^{1}$ L. Gross, J. Math. Phys. 5, 687-95 (1964).

${ }^{2}$ C. Boyer, E. G. Kalnins, and W. Miller, Jr., Nagoya

Math. J. 60, 35-80 (1976).

${ }^{3}$ E. G. Kalnins and W. Miller, Jr., J. Math. Phys. 17, 331

(1976).

${ }^{4}$ E. G. Kalnins and W. Miller, Jr., J. Math. Phys. 17, 369 (1976).

${ }^{5}$ E. G. Kalnins, W. Miller, Jr., and P. Winternitz, SIAM J. Appl. Math. 30, 630-64 (1976).

${ }^{6}$ E. G. Kalnins and W. Miller, Jr., J. Math. Phys. 18, 1 (1977).

${ }^{7}$ C. Boyer, E. G. Kalnins, and W. Miller, Jr., J. Math. Phys. 16, 499-511 (1975).

${ }^{8}$ E.G. Kalnins and W. Miller, Jr., J. Math. Phys. 16, 2507-16 (1975).

${ }^{9}$ E. G. Kalnins and W. Miller, Jr., J. Math. Phys. 17, 356 (1976).

${ }^{10} \mathrm{G}$. Post, J. Math. Phys. 17, 24-32 (1976).

${ }^{11} \mathrm{H}$. Bateman, Electrical and Optical Wave-Motion (Dover, New York, 1955), reprint of 1914 ed.

${ }^{12} \mathrm{H}$. Bateman, Partial Differential Equations of Mathematical Physics (Cambridge U. P., Cambridge, 1969), reprint of 1932 ed.

${ }^{13}$ H. Kastrup, Phys. Rev. 140, B183 (1965).

${ }^{14} \mathrm{~W}$. Miller, Jr., Symmetry Groups and Their Applications
(Academic, New York, 1972).

${ }^{15}$ W. Miller, Jr., Symmetry and Separation of Variables (Addison-Wesley, Reading, Mass., to appear).

${ }^{16}$ P. Dirac, Proc. Cambridge Philos. Soc. 30, 150-63 (1934).

${ }^{17}$ A. Davydov, Quantum Mechanics (Addison-Wesley, Reading, Mass., 1965).

${ }^{18}$ W. Miller, Jr., J. Math. Phys. 13, 1393-99 (1972).

${ }^{19}$ A. Makarov, J. Smorodinsky, K. Valiev, and P. Winternitz, Nuovo Cimento A 52, 1061-84 (1967).

${ }^{20}$ N. Ya. Vilenkin, Special Functions and the Theory of Group Representations (AMS Trans., Am. Math. Soc., Providence, R. I. , 1968).

${ }^{21}$ G. Burdet, M. Perrin, and P. Sorba, Commun. Math. Phys. 34, 85-90 (1973).

${ }^{22}$ C. Boyer, Helv. Phys. Acta 47, 589 (1974).

${ }^{23} \mathrm{~S}$. Helgason, Differential Geometry and Symmetric Spaces (Academic, New York, 1962).

${ }^{24}$ T. Hirai, Proc. Jpn. Acad. 42, 907-12 (1966).

${ }^{25} \mathrm{M}$. Bocher, Die Reihentwickelungen der Potentialtheorie (Leipzig, 1894).

${ }^{26}$ L. Eisenhart, Continuous Groups of Transformations (Dover, New York, 1961).

${ }^{27} \mathrm{H}$. Zassenhaus, Can. Math. Bull. 1, 31-39, 101-11, 183-91 (1958). 